\title{
POINTWISE ESTIMATE FOR LINEAR COMBINATIONS OF PHILLIPS OPERATORS
}

\author{
GANCHO TACHEV
}

Abstract. For pointwise approximation of bounded continuous functions by linear combinations of Phillips operators we represent equivalent relation by means of Ditzian-Totik modulus of smoothness. The rate of approximation is better compared with similar estimates, proved in the past for other Szász-type operators.

Mathematics subject classification (2010): 41A10, 41A25, 41A36.

Keywords and phrases: Pointwise approximation, Ditzian-Totik modulus of smoothness, linear combinations, inverse theorems, Phillips operators.

\section{REFERENCES}

[1] P. N. Agrawal And V. Gupta, On the iterative combinations of Phillips operators, Bull. Inst. Math. Acad. Sinica, 18, 4 (1990), 361-368.

[2] P. N. Agrawal And V. Gupta, On convergence of derivatives of Phillips operators, Demonstratio Mathematica, 27, 2 (1994), 501-510.

[3] P. N. Agrawal, V. Gupta And A. R. Gairola, On derivatives of an iterative combinations of Phillips operators, Nonlinear Functional Analysis and Applications, 12, 2 (2007), 195-203.

[4] J. Bustamante, J. Quesada And L. CRUZ, Direct estimate for linear operators in polynomial weighted spaces, J. Approx. Theory, 162 (2010), 1495-1508.

[5] P. L. Butzer, Linear combinations of Bernstein polynomials, Canad. J. Math., (1953), 559-567.

[6] V. Gupta And P. N. Agrawal, Linear Combinations of Phillips Operators, Indian Acad. Math., 11, 2 (1989), 106-114.

[7] V. Gupta And P. N. AgRawal, Lp-Approximation by iterative combination of Phillips operators, Publ. de l'Institut Mathematique, 52 (66), (1992), 67-76.

[8] V. Gupta And A. SAHAi, On the linear combinations of Phillips operators, Soochow Journal of Mathematics, 19, 3 (1993), 313-323.

[9] S. Guo, C. Li, Y. Sun, G. Yang And S. Yue, Pointwise Estimate for Szász-Type Operators, J. Approx. Theory, 94 (1998), 160-171.

[10] S. Guo, S. Yue, C. Li, G. Yang And Y. Sun, A pointwise approximation theorem for linear combinations of Bernstein polynomials, Abstr. Appl. Anal., 1 (1996), 359-368.

[11] S. Guo, C. Li And X. LiU, Pointwise Approximation for Linear Combinations of Bernstein Operators, J. Approx. Theory, 107 (2000), 109-120.

[12] S. Guo, L. LiU And Q. QI, Pointwise Estimate for Linear Combinations of Bernstein-Kantorovich Operators, J. Math. Anal. Appl., 265 (2002), 135-147.

[13] Z. DitZIAN, A global inverse theorem for combinations of Bernstein polynomials, J. Approx. Theory, 26 (1979), 277-292.

[14] Z. Ditzian And V. TotiK, Moduli of Smoothness, Spriner Verlag, New York, (1987).

[15] Z. Ditzian AND K. Ivanov, Strong converse inequalities, J. Anal. Math., 61 (1993), 61-111.

[16] M. Heilmann, Erhöhung der Konvergenzgeschwindigkeit bei der Approximation von Funktionen mit Hilfe von Linearkombinationen spezieller positiver linear Operatoren, Habilitationsschrift, Universität Dortmund, (1991).

[17] M. Heilmann, Approximation auf $[0, \infty)$ durch das Verfahren der Operatoren vom BaskakovDurrmeyer Typ, Dissertation, Universität Dortmund, (1987). 
[18] M. Heilmann And G. TAChev, Commutativity, direct and strong converse results for Phillips operators, East Journal on Approximations, 17, 3 (2011), 299-317.

[19] Margareta Heilmann And Gancho TACHeV, Linear Combinations of Genuine Szasz-Mirakjan - Durrmeyer Operators, Springer Proceedings in Mathematics and Statistics with Volume title Advances in Applied Mathematics and Approximation Theory - Contributions from AMAT 2012 Conference, Turkey, ed. by G. Anastassiou and O. Duman, (2012), 5-th Chapter, 85-106.

[20] C. P. MAY, On Phillips Operator, J. Approx. Theory, 20, (1977), 315-322.

[21] S. M. Mazhar And V. Totik, Approximation by modified Szász operators, Acta Scientiarum Mathematicarum, 49, (1985), 257-269.

[22] R. S. PHILlips, An inversion formula for Laplace transforms and semi-groups of linear operators, Annals Math. 59, 2 (1954), 325-356.

[23] GANCHO TACHEV, Approximation of bounded continuous functions by linear combinations of Phillips operators, Demonstratio Mathematica, XLVII, 3 (2014), 662-671.

[24] Gancho Tachev, A Global Inverse Theorem for Combinations of Phillips Operators, accepted in Mediterranean Journal of Mathematics, (2015).

[25] LINSEN XIE, The lower estimate for the linear combinations of Bernstein-Kantorovich operators, J. Approx. Theory, 162, (2010), 1150-1159.

[26] Linsen XIE, Pointwise simultaneous approximation by combinations of Bernstein operators, J. Approx. Theory, 137, (2005), 1-21.

[27] LinsEn XIE, Strong type of Stechkin inequality for the linear combination of Bernstein operators, J. Math. Anal. Appl., 408, (2013), 615-622.

[28] D. X. Zhou, On a Paper of Mazhar and Totik, J. Approx. Theory, 72, (1993), 290-300. 